\title{
A comparison of the chicken and turkey proteomes and phosphoproteomes in the development of poultry-specific immuno-metabolism kinome peptide arrays
}

\author{
Ryan J. Arsenault ${ }^{1}{ }^{*}$, Brett Trost $^{2}$ and Michael H. Kogut ${ }^{1}$ \\ 1 United States Department of Agriculture, Southern Plains Agricultural Research Center (SPARC), Agricultural Research Service, College Station, TX, USA \\ 2 Department of Computer Science, University of Saskatchewan, Saskatoon, SK, Canada
}

Edited by:

Maureen T. Long, University of Florida College of Veterinary Medicine, USA

\section{Reviewed by:}

Olivia Bowen Faulkner, University of Arkansas, USA

Damer Blake, Royal Veterinary

College, UK

\section{*Correspondence:}

Ryan J. Arsenault, United States Department of Agriculture, Southern Plains Agricultural Research Center (SPARC), Agricultural Research Service, 2881 F\&B Road, College Station, TX 77845, USA

e-mail: ryan.arsenault@usask.ca
The use of species-specific peptide arrays for the study of animal kinomes has a proven track record of success. This technique has been used in a variety of species for the study of host-pathogen interactions and metabolism. Species-specific peptide arrays have been designed previously for use with chicken but a turkey array has never been attempted. In addition, arrays designed around individual cellular functions have been designed and utilized, but cross-function immuno-metabolic arrays have not been considered previously. Antecedent to designing separate chicken and turkey immuno-metabolic kinome peptide arrays, we show that while the chicken and turkey genomes are quite similar, the two species are much more distinct at the proteome and phosphoproteome levels. Despite a genome identity of approximately $90 \%$, we observe that only $83 \%$ of chicken and turkey orthologous proteins display sequence matches between the two species. Further, less than $70 \%$ of kinase recognition target sequences are exact matches between chicken and turkey. Thus, our analysis shows that, at the proteome and kinome level, these two species must be considered separately in the design of novel peptide arrays. Our ultimate array design covers numerous immune and metabolic processes including innate and adaptive immunity, inflammatory responses, carbohydrate, protein, and fat metabolism, and response to hormones. We have shown the proteomic and phosphoproteomic diversity of chicken and turkey and have designed a valuable research tool for the study of immuno-metabolism within these two species.

Keywords: kinome, chicken, turkey, poultry, peptide array, phosphorylation, proteome, immuno-metabolism

\section{INTRODUCTION}

Genomics and genetics are dominant approaches in the study of physiology and disease states across species. The tools within these fields have advanced substantially and enable the rapid collection of significant amounts of data; this now includes the rapid sequencing of entire species genomes (1). While sequencing the complete genome of an organism is an important means of biological understanding and discovery, it can be very difficult to translate this information into individual host responses due to stimulation or treatment, and ultimate organism phenotype. Transcriptomics, being a more focused branch of genetics, allows one to consider only the expressed genes converted to mRNA. There are a wide variety of tools available for numerous species to study the transcriptome. The downside of transcriptomic approaches, especially when attempting to determine final phenotype, is that there are several processes and potential disruptions that can occur before the final active protein is generated. These include gene silencing, mRNA stability, translation, translational efficiencies, protein turnover, sequestration of enzymes from substrates, and the multitude of post-translation modifications, of which phosphorylation is a major class.

Studying physiology and organism response at the proteomelevel allows one to consider biology near or at the final phenotype.
The active proteins are the ultimate effectors that influence phenotype. Within proteomics studying the enzymatic activity of a protein allows one to consider the final step in a cellular response. Kinomics is the study of kinase enzymes activated under given conditions within a cell or tissue. Kinases are enzymes that phosphorylate proteins, changing the target protein's activity in some way (2). Cellular signal transduction through protein phosphorylation is a post-translational modification that plays a role in the regulation of nearly every cellular process and function (3); thus, the study of kinomics can provide insight into the various cellular processes at an active protein level. In this type of approach, all of the intervening steps between gene and phenotype, such as gene silencing, the effects of non-coding RNA, and improper protein folding and function, are eliminated.

The technique described here to study the cellular kinome is peptide microarrays. There are other techniques that have been used to study the kinome including phospho-antibodies and massspectrometry $(4,5)$. Peptide arrays have a number of advantages including the availability of reagents and equipment, the ability to focus only on the active kinases within a sample, and the ability to tailor the analysis to particular species, cellular functions, and individual phosphorylation sites (6). Peptide arrays have been used to study the kinomics of cellular biology $(7,8)$ and host-pathogen 
interactions (9-13). One of the key peptide array technology developments was the establishment of species-specific peptide arrays, providing a tool for the study of veterinary species at the kinome level (14).

A species-specific peptide array allows for the study of the kinome of nearly any species. The process of designing a speciesspecific array begins with the known phosphorylation sites within the species of interest, as well as a database of phosphorylation sites from an evolutionarily close species (14). While some species such as human, mouse, rat, and fruit fly have relatively well-annotated proteomes and phosphoproteomes, other species such as chicken, turkey, sheep, and honey-bee are less well-studied. Within the custom-designed software pipeline DAPPLE (15), one uses databases of experimentally determined phosphorylation sites from other organisms to query the complete proteome of the species of interest for orthologous phosphorylation sites. Using DAPPLE, it is possible to determine if a kinase target sequence from the query species is present and located within the orthologous protein of the target species. Using these orthologous kinase target peptides, it is possible to design a species-specific kinome peptide array. This technique has been used to design species-specific peptide arrays for species as diverse as cow $(9,11)$, chicken (10), pig (16), and honey bee (17) for cellular functions from innate immunity (18) to metabolism (10). The primary advantage of using species-specific arrays is one does not have to rely on potential cross-reactions between a commercially available peptide array (usually human or mouse) and the species of interest. The kinase target sites printed onto the array are designed to be exact matches to the proteome of the given species. The limited homology in human peptide arrays for the study of other species (19) as well as the lack of cross-reactivity or unexpected cross-reactivity observed in species-specific antibodies (20) illustrates the fact that one cannot rely on cross-species cross-reactivity at the level of peptide recognition. Our own analysis has shown that the species variation is significantly greater at the phosphorylation target site sequence than at the genome level (6). Here, we show that this is true when comparing chicken and turkey phosphorylation target sequences as well.

The chicken (Gallus gallus) and turkey (Meleagris gallopavo) diverged approximately 40 million years ago (21). Despite this length of separate evolution, the genomes of chicken and turkey are relatively similar. As a comparison, rhesus macaque and humans separated 25 million years ago, and these species display significantly greater genome differences than chicken and turkey (21). An Ensembl LastZ alignment between the human and macaque genomes show an $80 \%$ complete genome coverage, while the chicken and turkey genomes show an $89 \%$ complete genome coverage. Other avian genomes align significantly as well; a three-way alignment between chicken, turkey, and zebra finch shows alignments of 91.92, 92.39, and $81.51 \%$, respectively (21).

Though the genomes of chicken and turkey appear relatively similar, more specific genetic study reveals evidence of why we cannot simply assume orthology. In the case of the immune systems of chickens and turkeys, species diversity is a key consideration. Genomic evidence involving the innate immune system points to stronger positive selection on these genes between chicken and turkey than between other species pairs including chicken-zebra finch and turkey-zebra finch (21). When comparing chicken to turkey, the level of positive selection of the innate immune genes (as measured by $\mathrm{dN} / \mathrm{dS}$ ratio) approaches the level of non-immune genes (21). This level of positive selection would not be expected in a set of genes normally undergoing purifying selection. This result indicates that innate immune genes of these two species will be more evolutionarily divergent than would be anticipated, further strengthening the argument for a species-specific approach. Here, we have taken this comparative approach to the level of the proteome and phosphoproteome to understand the level of divergence between the chicken and turkey within the key physiological processes of immunity and metabolism.

The interface of the immune system and metabolism is an emerging field of study (22). The initial impetus for researchers to consider an immuno-metabolic perspective was the human health concerns related to obesity, diabetes, and metabolic disorder. Excessive fat deposition can lead to an innate immune inflammatory response. This chronic low-grade inflammation was linked to resultant diseases such as type 2 diabetes, fatty liver disease, and cirrhosis (22). Later, some of the classical metabolic energy sensors and energy switches, such as AKT1-3, AMPK, mTOR, and LKB1, were shown to be linked to $\mathrm{CD}^{+} \mathrm{T}$ cell functions (23). From there, links between metabolism, immunity, and host response to infectious disease grew. Within animal agriculture, and poultry production specifically, a consideration of the immuno-metabolic consequences will be invaluable. A focus solely on maximizing animal growth can reduce immune potential, while a strong immune response has negative consequences on growth (24). A consideration of the integrated whole may allow one to maximize growth and animal production without having a detrimental impact on animal health and immune potential. In this study, we show the nearly innumerable links between cellular signaling proteins classically characterized as members of either the immune or metabolic functional groups. Due to these links, we feel an integrated immuno-metabolic approach would be a valuable research perspective; thus, we describe here for the first time the design of chicken and turkey species-specific, immuno-metabolic peptide arrays for kinome analysis, based on the proteomic analysis of these two species.

\section{MATERIALS AND METHODS IDENTIFICATION OF PUTATIVE CHICKEN AND TURKEY PHOSPHORYLATION SITES}

The DAPPLE $(15)^{1}$ software pipeline was used to identify phosphorylation sites in the chicken and turkey proteomes that were orthologous to experimentally determined phosphorylation sites from other organisms. The PhosphoSitePlus database $(25)^{2}$ was used as the source of experimentally determined phosphorylation sites. At the time of analysis, it contained 229,173 such sites, most of which were from human, mouse, rat. Each of these sites was represented as a 15-mer kinase target sequence, with the potential phosphorylation residue in the center and seven residues on either side. In cases where the phosphorylation residue was too

\footnotetext{
${ }^{1}$ http://saphire.usask.ca/saphire/dapple/index.html

${ }^{2}$ http://www.phosphosite.org
} 
close to the $\mathrm{N}$ - or C-terminus of the full protein for this to be possible, the phosphorylation site was represented by the first 15 residues or last 15 residues of the full protein, respectively. For each of these peptides, DAPPLE performed a protein BLAST search using the peptide as the query, and the chicken or turkey proteome as the database. DAPPLE then reports the best match in the chicken or turkey proteome for that 15-mer peptide, as well as additional information that facilitates the selection of peptides to include on an array (for example of DAPPLE output see Table S2 in Supplementary Material).

\section{PEPTIDE SELECTION AND ARRAY DESIGN}

Once the list of putative chicken and turkey phosphorylation target sites were generated by DAPPLE, the individual peptides containing phosphorylation sites were selected for incorporation into the final array. This selection was based both on the design goals of the array, and on the confidence that a given chicken or turkey site identified by DAPPLE was a true phosphorylation site. With respect to the design goals of the array, the intention was to design an immuno-metabolic peptide array. As such, peptides were selected that derived from proteins falling into one of three categories: (1) proteins that could be considered central cellular signaling hubs (for example, AKT, MAPK, PI3K); (2) proteins involved in the innate and adaptive immune systems; and (3) proteins involved in metabolic processes (for example, glycolysis, fatty acid synthesis, protein catabolism, protein synthesis). With respect to the confidence that a given peptide identified by DAPPLE indeed contained a phosphorylation site, several criteria were considered. First, DAPPLE outputs the number of sequence differences between the query 15-mer and its best match in the chicken or turkey proteome. Peptides for which the number of sequences differences was small were preferentially selected for inclusion on the array. Second, DAPPLE determines whether the full protein corresponding to the query peptide, and the full protein corresponding to the query peptide's closest match in the chicken or turkey proteome, are reciprocal BLAST hits (and thus are likely to be orthologs). We preferentially selected putative chicken or turkey phosphorylation sites for which the corresponding proteins were reciprocal BLAST hits. Third, DAPPLE outputs the phosphorylated residue in the corresponding full protein (e.g., Y25) for both the query phosphorylation site and its best hit. Putative chicken or turkey sites for which the position of the phosphorylated residue was similar to the query site were preferred.

\section{CHARACTERIZATION OF PROTEOME-LEVEL CONSERVATION BETWEEN CHICKEN AND TURKEY}

To determine the level of conservation between chicken and turkey at the proteome level, a list of orthologous proteins between the two species was downloaded from OrthoDB $(26)^{3}$. For each pair of orthologs, the EMBOSS (27) program needle (28) was used to determine the optimal global alignment between the two proteins, as well as the \% identity and the percentage of alignment positions that contained gaps.

\footnotetext{
${ }^{3}$ http://cegg.unige.ch/orthodb6
}

\section{CHARACTERIZATION OF KINASE TARGET SEQUENCE CONSERVATION BETWEEN HUMAN AND OTHER SPECIES}

The PhosphoSitePlus database (25) was filtered to include only phosphorylation sites from human. Using DAPPLE, the 15-mer peptides corresponding to these sites were used as BLAST queries against four target proteomes (chimpanzee, mouse, chicken, and turkey). The number of sequence differences between a given query peptide and its best match in the target proteome was recorded.

\section{CHARACTERIZATION OF KINASE TARGET SITE CONSERVATION BETWEEN CHICKEN AND TURKEY}

Because few phosphorylation sites have been experimentally characterized in chicken, and none are known in turkey, it is difficult to directly assess the level of phosphorylation site conservation between these species. As such, the following procedure was performed to estimate the level of kinase target site 15-mer conservation. Using DAPPLE, the 15-mer peptide corresponding to each of the known phosphorylation sites in the entire PhosphoSitePlus database (25) was searched against the chicken proteome. All of the matches in the chicken proteome that had seven or fewer sequence differences from the known 15-mer peptide sequence were then used as input to DAPPLE, this time with the turkey proteome as the target. The number of sequence differences between a given chicken sequence, and its best match in the turkey proteome, was then recorded. This process was then repeated in reverse, with the contents of PhosphoSitePlus initially being searched against the turkey proteome, and then the matches with seven or fewer sequence differences being searched against the chicken proteome.

\section{RESULTS}

At the genome and gene level, the chicken and turkey show significant similarity. Genome coverage between the two species is $89 \%$, while exon coverage is $90 \%$ (Ensembl LastZ alignment). Coverage represents the percentage of bases in the shorter of the input sequences that align. Pecan alignment of the chicken, turkey, and zebra finch genomes shows that chicken and turkey align at 91.92 and $92.39 \%$, respectively (21). However, this level of genetic similarity does not translate to the protein level. The online database OrthoDB (26) lists 9,816 putative orthologs between chicken and turkey. As the chicken proteome contains approximately 23,000 proteins and the turkey proteome contains approximately 16,000 proteins (29), both chicken and turkey encode many proteins for which an ortholog does not exist in the other species. With respect to the proteins that do have orthologs in the other species, the average percent identity between these pairs of orthologs was $83.3 \%$, with gaps in the alignment accounting for $12.4 \%$ of the difference (Table 1). As such, the level of similarity between chicken and turkey was lower at the protein level than at the genome level, and it is worth recalling that this level of difference is in the proteins that have been determined to be orthologous.

In the design of species-specific peptide arrays, the sequences of interest between orthologous proteins is the kinase target recognition sequence. These are sequences of 15 amino acids containing either a serine, threonine, or tyrosine central residue that is recognized by a specific kinase and phosphorylated (30). As a first step in analyzing the kinase target 15-mers, we utilized 
Table 1 | Comparison of chicken to turkey protein sequence identity.

\begin{tabular}{lcccc}
\hline & $\begin{array}{l}\text { \% Sequence } \\
\text { identity }\end{array}$ & $\begin{array}{l}\text { \% Due to } \\
\text { sequence } \\
\text { gaps }\end{array}$ & Total \% & $\begin{array}{l}\text { No. of } \\
\text { protein } \\
\text { pairs }\end{array}$ \\
\hline $\begin{array}{l}\text { Ortholog-to-ortholog } \\
\text { comparison }\end{array}$ & 83.28 & 12.37 & 95.65 & 9816 \\
\hline
\end{tabular}

Ortholog-to-ortholog comparisons are only those proteins shown to be orthologous based on OrthoDB (26). \% Sequence identity indicates sequence similarity between pairs of proteins and \% sequence gaps is the \% of the protein that failed to align to due gaps in the sequence as opposed to amino acid mismatches.

the large and well-annotated PhosphoSitePlus online database (25). At the time of analysis, this database contained 229,173 such phosphorylation target sequences. To determine the level of conservation of phosphorylation sites among different organisms, the human sequences from this database were used to query the proteomes of chicken, turkey, mouse, and chimpanzee using the DAPPLE software platform (15). This analysis provided us with both an indication of the relative similarities of kinase target sequences between each species and human, and a relative level of similarity between chicken and turkey within this portion of the proteome. As expected, the chimpanzee was most similar to human in phosphorylation site conservation, with $82.98 \%$ of the 15-mer queries being identical between human and chimpanzee (Table 2). This was a significantly greater percentage than mouse, chicken, or turkey, but it is much less than the sequence identity observed between the human and chimpanzee genomes (31). Of the four species analyzed, mouse was the next most similar to human beings, which was expected considering both are mammals. Finally, comparing human 15-mer peptides to chicken and turkey indicated that these two species are relatively similar in their distance from human: $16.20 \%$ of sequences exhibited complete identity in chicken vs. $15.15 \%$ in turkey. Conversely, approximately $40 \%$ of the human 15 -mers had very weak matches (seven or more sequence differences) in both the chicken and turkey proteomes.

Following the comparison of phosphorylation site conservation between human and each of mouse, chicken, and turkey, we compared the 15-mer sequences in chicken (as predicted by DAPPLE using all of the entries in PhosphoSitePlus as queries) to the turkey proteome, and vice-versa. In the chicken to turkey comparison, $69.19 \%$ of putative chicken phosphorylation sites had exact matches in the turkey proteome (Table 3 ). In the reverse comparison, $75.61 \%$ of turkey 15 -mers had exact matches in the chicken proteome. The difference in percentage of exact matches can likely be attributed to the proteome sizes of the two species. Given that the chicken proteome (approximately 23,000 proteins) is significantly larger than the turkey proteome (approximately 16,000 proteins) (29), it was not unexpected that a higher percentage of turkey peptides would have exact matches in the chicken proteome compared to the reverse.

The level of phosphorylation site conservation between chicken and turkey suggests that it would be possible to design a single array that is specific to both chicken and turkey. Given the percentages reported in Table 2, there are tens of thousands of peptides that are found as exact matches in both the chicken and turkey proteomes,
Table 2 | Conservation between human kinase target sequences and those of various other species.

\begin{tabular}{lcccc}
\hline $\begin{array}{l}\text { Sequence } \\
\text { differences }\end{array}$ & \multicolumn{4}{c}{ Peptides vs. human (out of 146,765) } \\
\cline { 2 - 5 } & \multicolumn{4}{c}{} \\
\cline { 2 - 5 } & Chimpanzee (\%) & Mouse (\%) & Chicken (\%) & Turkey (\%) \\
\hline 0 & 82.98 & 36.67 & 16.20 & 15.15 \\
1 & 7.21 & 17.41 & 10.29 & 9.74 \\
2 & 1.38 & 11.34 & 8.63 & 8.33 \\
3 & 0.64 & 7.77 & 7.31 & 7.26 \\
4 & 0.51 & 5.71 & 6.27 & 6.09 \\
5 & 0.59 & 4.45 & 5.85 & 5.72 \\
6 & 0.96 & 4.11 & 7.41 & 7.35 \\
$7+$ & 5.72 & 12.54 & 38.04 & 40.36 \\
\hline
\end{tabular}

Known human phosphorylation sites were obtained from PhosphoSitePlus (25) and were searched using DAPPLE (15) against each animal proteome. Sequences differences are out of the 15 total amino acids per sequence and \% of peptides are the number of peptides out of the total analyzed that had the indicated number of differences within its 15-mer.

Table 3 | Conservation between chicken and turkey phosphorylation target sites.

\begin{tabular}{lcc}
\hline & Chicken to turkey & Turkey to chicken \\
\cline { 2 - 3 } Total peptide sequences & $\mathbf{1 7 0 , 7 0 9}$ & $\mathbf{1 6 5 , 6 4 7}$ \\
\hline Sequence differences & \% of peptides & \% of peptides \\
\hline 0 & 69.19 & 75.61 \\
1 & 10.20 & 10.94 \\
2 & 3.68 & 3.31 \\
3 & 1.90 & 1.55 \\
4 & 1.37 & 1.06 \\
5 & 1.30 & 1.00 \\
6 & 1.78 & 1.23 \\
$7+$ & 10.58 & 5.31 \\
\hline
\end{tabular}

Total peptide sequences refer to the total number of amino acid sequences within the first species' proteome. Sequences differences are out of the 15 total amino acids per sequence and\% of peptides are the number of peptides out of the total analyzed that had the indicated number of differences within its 15-mer.

and thus that could potentially be selected for inclusion on an array that is equally applicable to chicken and turkey. Nonetheless, it is often desirable to include very specific peptides of interest on the array. When we consider that the genetic homology between these two species is approximately $90 \%$, we see that there are significant differences between these two species at the proteome level that must be considered in any proteomic analysis. As such, it is not necessarily possible to create an array that is equally applicable to both turkey and chicken, as some of the kinase target sequences the researcher wants represented may have sequence differences between chicken and turkey.

We have previously used chicken-specific peptide arrays designed to study immune response (32) and metabolic changes (10). Using the STRING protein-protein interaction 


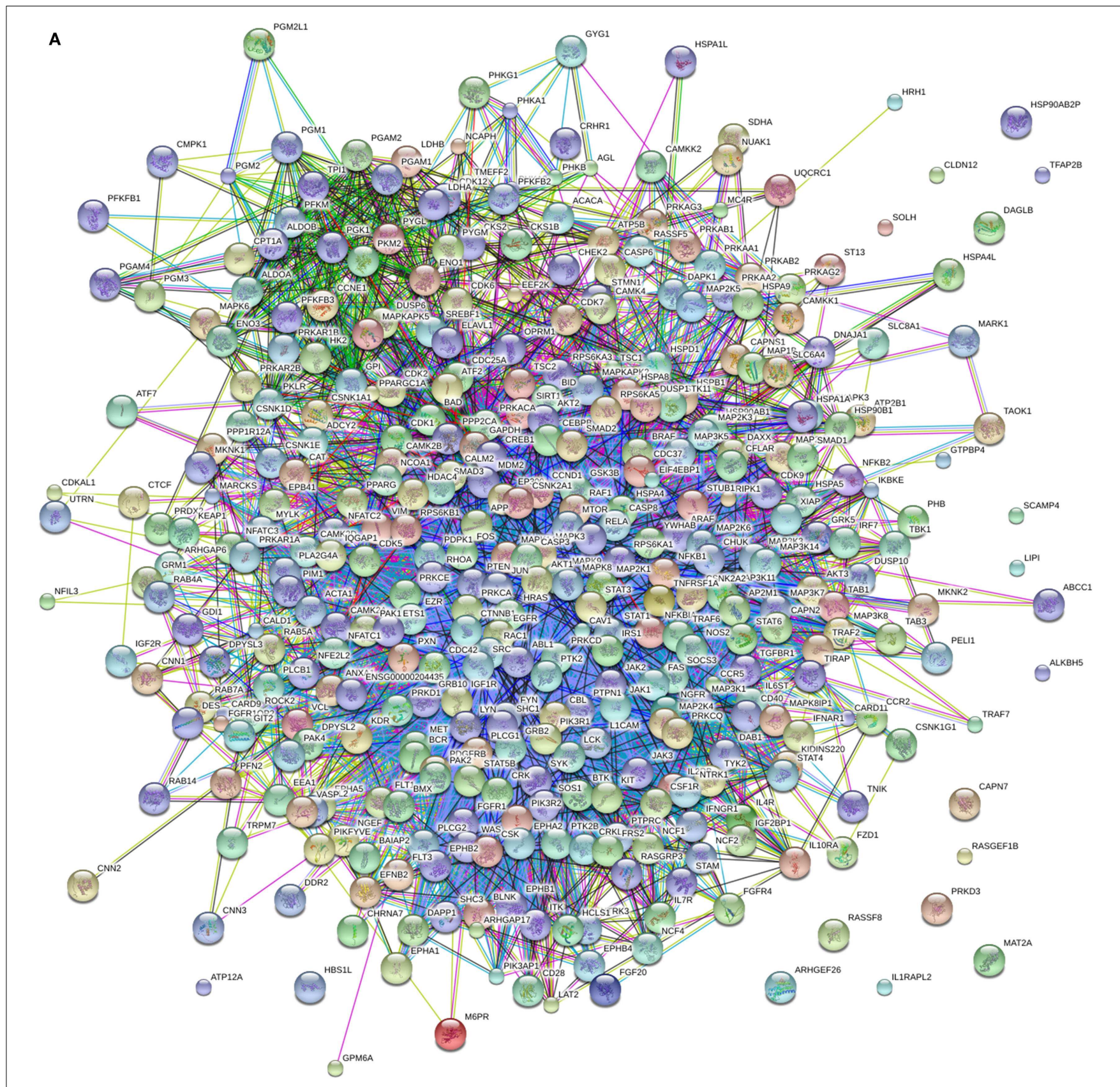

FIGURE 1 | STRING output of interactions between proteins of the original chicken immune and metabolic peptide arrays. (A) Protein-protein interactions between proteins included in the immune peptide array and metabolic peptide array.

(Continued)

database $(33)^{4}$, we looked into the potential links between proteins represented on these two functionally distinct arrays. Figure 1A shows the complete network of interactions generated from the unique proteins represented by the two arrays. Using the GO term Biological Process categorization feature of STRING, we were able to highlight the proteins within the network that

${ }^{4} \mathrm{http}: / /$ string-db.org corresponded to immune processes (Figure 1B) and metabolic processes (Figure 1C). These figures show that proteins from the two distinct biological process categories do not show up as distinct nodes (i.e., separated physically in the figure), but are interspersed and linked to one another in a number of ways. Based on the number of connections between the "immune" proteins and the "metabolism" proteins, it could be argued that these protein groupings are not distinct at all; thus, we have the rationale for the design of the first-ever species-specific kinome peptide array for the study 


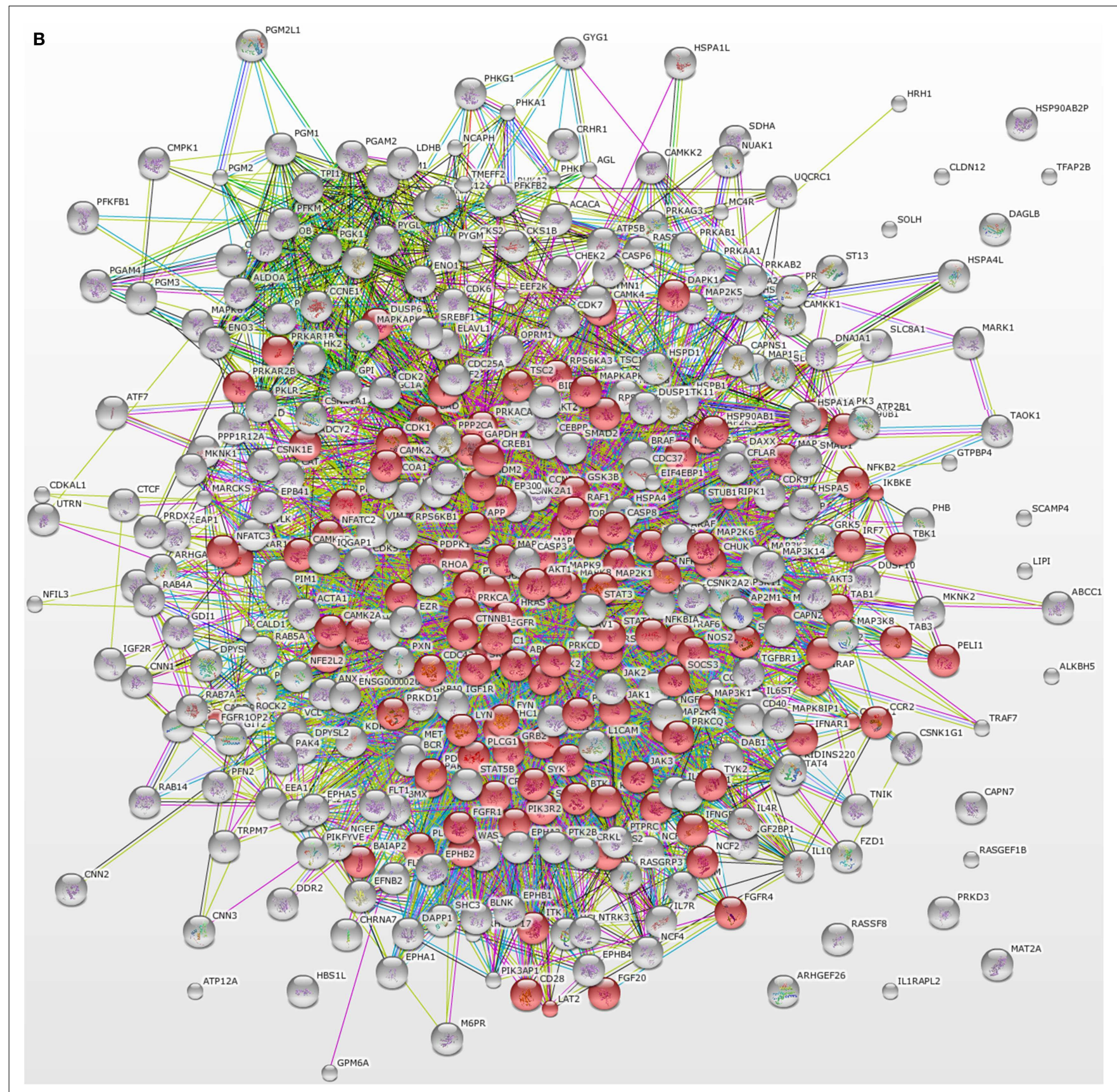

FIGURE 1 | (B) Red circles indicate immune-related proteins based on GO Biological Function terms.

(Continued)

of the integrated immuno-metabolomic responses within the two poultry species.

The ideal design for an immuno-metabolic array would incorporate as large a cross section of the two biological processes as possible, selected from the broad immune and metabolism categories. These include fatty acid, carbohydrate and protein metabolism, innate and adaptive immunity, hormonal metabolism and response, and key signaling molecules that cover the central signaling hubs of key pathways. Peptides representing proteins' kinase target sites from these groups were individually selected from the DAPPLE output to be included on the combined immuno-metabolic array. Table 4 provides a breakdown of the number of peptides on the final array and the biological processes in which they are involved. Since many of the proteins on the array are involved in more than a single process, there are a large number of proteins listed within Table 4 . The fact that many proteins are involved in several different metabolism- and immune-related biological processes emphasizes the numerous 


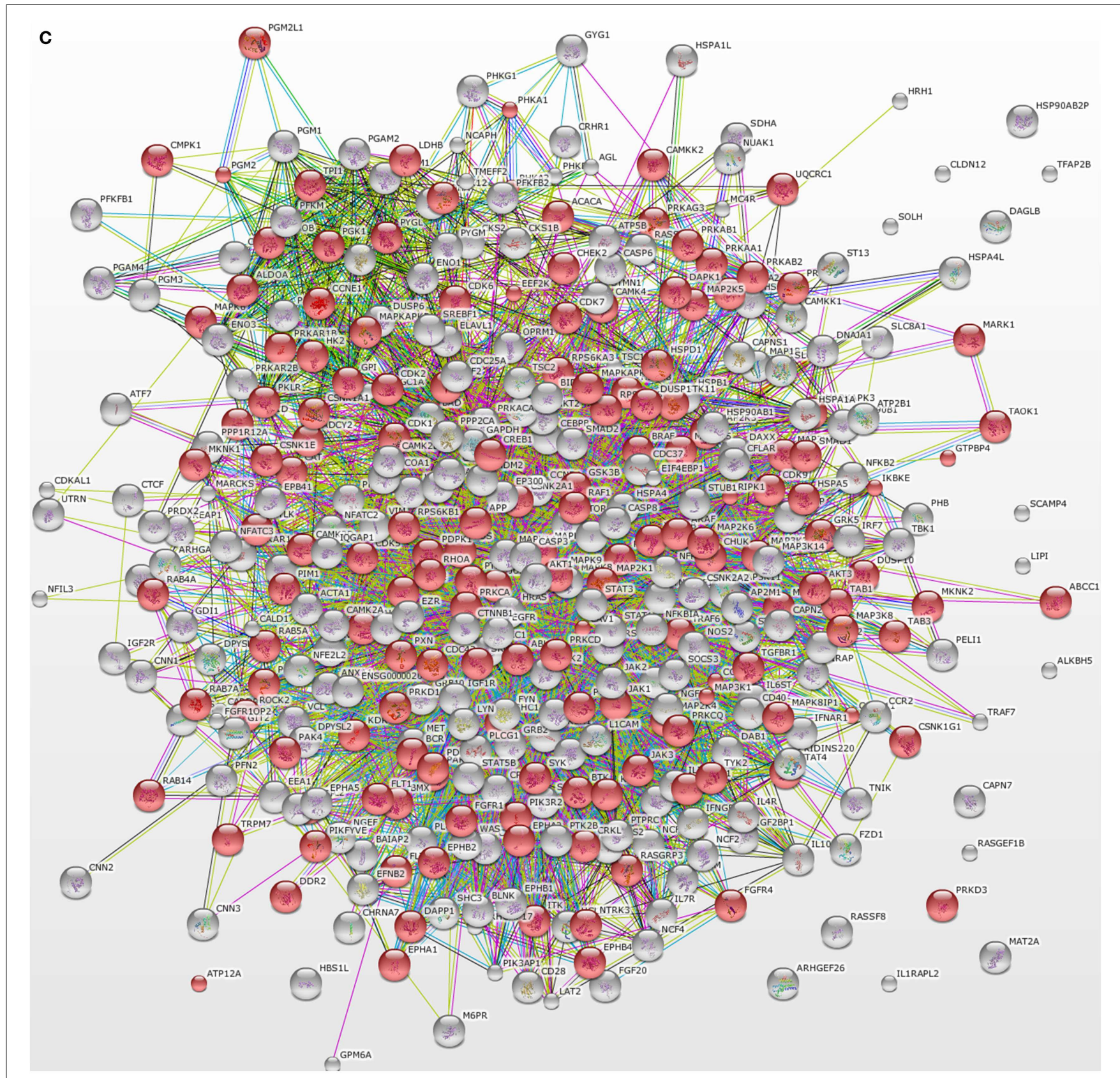

FIGURE 1 | (C) Red circles indicate metabolism-related proteins based on GO Biological Function terms. The figure illustrates the numerous interactions between the immune and metabolism related proteins.

links between immune and metabolic signaling (as observed in Figure 1).

As the proteome analysis indicated, significant differences in the proteomes of chicken and turkey, and specifically within the kinase recognition sites of phosphorylated proteins, suggest that distinct chicken and turkey peptide arrays should be designed. With the level of proteome divergence, even within these evolutionarily close species, a cross-species chicken/turkey array would not take full advantage of the potential for maximum kinase recognition and signal detection possible with a species-specific array.
Table S1 in Supplementary Material includes the proteins, peptide sequences, accession numbers, and protein annotations for the chicken immuno-metabolic peptide array. Table S2 in Supplementary Material includes the DAPPLE output for the equivalent proteins within the turkey.

\section{DISCUSSION}

In general, genome sequencing and genetics have highlighted the remarkable similarity across species and have emphasized the evolutionary links among different species. However, logically, 
Table 4 | GO term biological processes incorporated into immuno-metabolic peptide array.

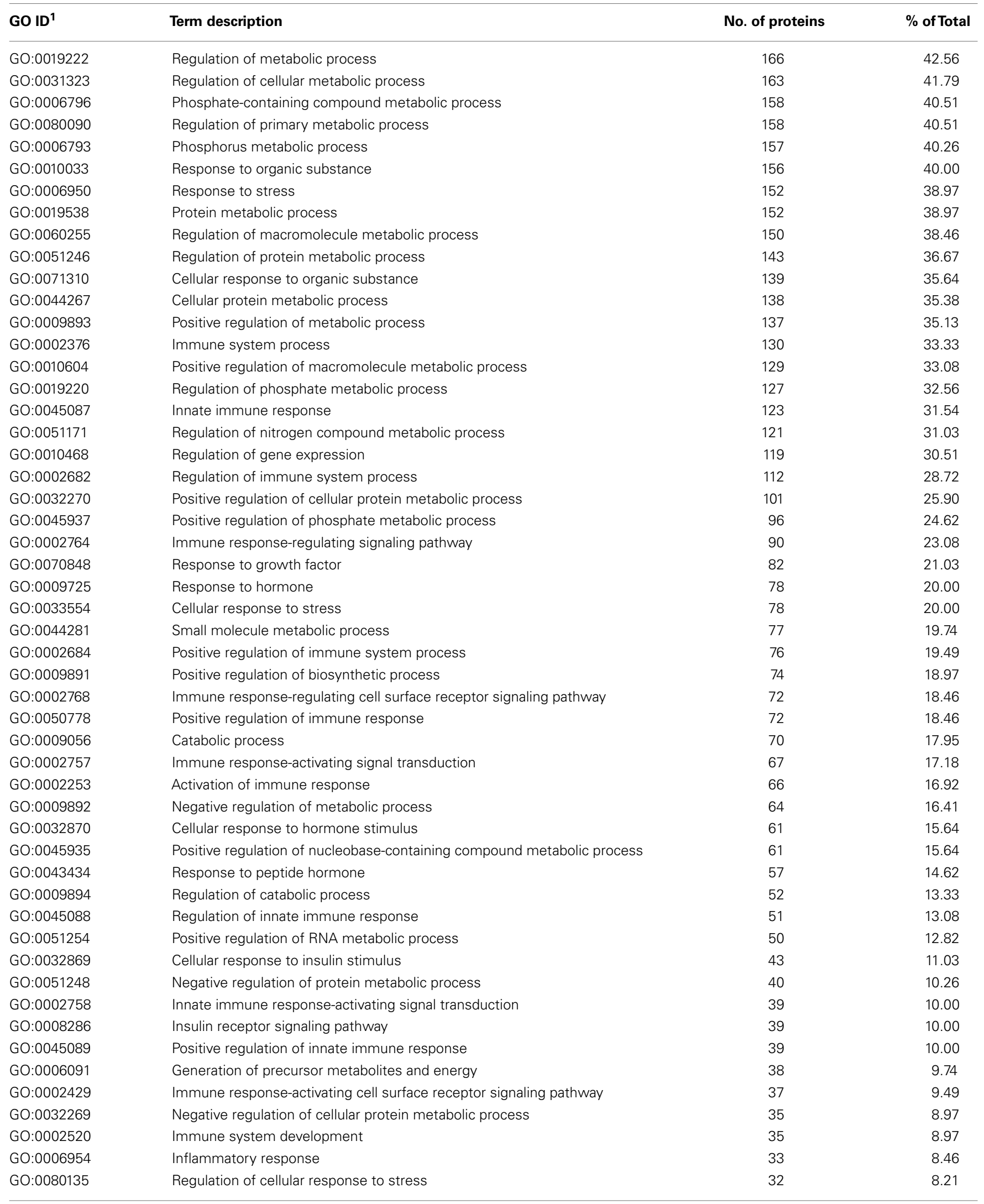


Table 4 | Continued

\begin{tabular}{|c|c|c|c|}
\hline GO ID ${ }^{1}$ & Term description & No. of proteins & $\%$ of Total \\
\hline GO:0006006 & Glucose metabolic process & 31 & 7.95 \\
\hline GO:0009617 & Response to bacterium & 31 & 7.95 \\
\hline GO:0048545 & Response to steroid hormone & 30 & 7.69 \\
\hline GO:0019318 & Hexose metabolic process & 28 & 7.18 \\
\hline GO:0005996 & Monosaccharide metabolic process & 28 & 7.18 \\
\hline GO:0019216 & Regulation of lipid metabolic process & 28 & 7.18 \\
\hline GO:0031667 & Response to nutrient levels & 24 & 6.15 \\
\hline GO:0016052 & Carbohydrate catabolic process & 21 & 5.38 \\
\hline GO:0002433 & Immune response-regulating cell surface receptor signaling pathway involved in phagocytosis & 21 & 5.38 \\
\hline GO:0060191 & Regulation of lipase activity & 20 & 5.13 \\
\hline GO:0009743 & Response to carbohydrate & 20 & 5.13 \\
\hline GO:0016051 & Carbohydrate biosynthetic process & 18 & 4.62 \\
\hline GO:0002819 & Regulation of adaptive immune response & 17 & 4.36 \\
\hline GO:0050864 & Regulation of B cell activation & 15 & 3.85 \\
\hline GO:0019217 & Regulation of fatty acid metabolic process & 14 & 3.59 \\
\hline GO:0006094 & Gluconeogenesis & 12 & 3.08 \\
\hline GO:0019319 & Hexose biosynthetic process & 12 & 3.08 \\
\hline GO:0046364 & Monosaccharide biosynthetic process & 12 & 3.08 \\
\hline GO:0060396 & Growth hormone receptor signaling pathway & 11 & 2.82 \\
\hline GO:0071378 & Cellular response to growth hormone stimulus & 11 & 2.82 \\
\hline GO:0005977 & Glycogen metabolic process & 11 & 2.82 \\
\hline GO:0044042 & Glucan metabolic process & 11 & 2.82 \\
\hline GO:0006073 & Cellular glucan metabolic process & 11 & 2.82 \\
\hline GO:0005980 & Glycogen catabolic process & 10 & 2.56 \\
\hline GO:0009251 & Glucan catabolic process & 10 & 2.56 \\
\hline GO:0044247 & Cellular polysaccharide catabolic process & 10 & 2.56 \\
\hline GO:0000272 & Polysaccharide catabolic process & 10 & 2.56 \\
\hline GO:0044275 & Cellular carbohydrate catabolic process & 10 & 2.56 \\
\hline GO:0006096 & Glycolysis & 10 & 2.56 \\
\hline GO:0060334 & Regulation of interferon-gamma-mediated signaling pathway & 9 & 2.31 \\
\hline
\end{tabular}

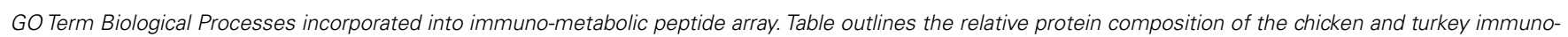

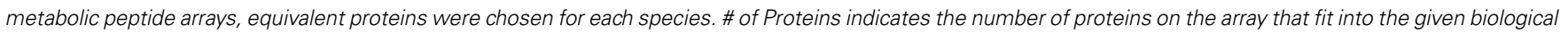

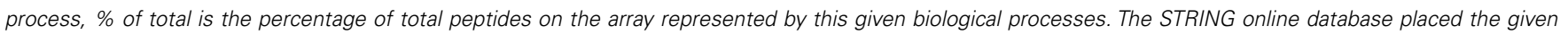
number of proteins into their GO Term categories following input of the peptide array protein list.

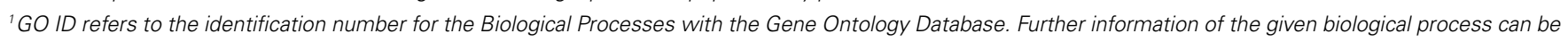
found by querying the databse using the GO ID number.

considering the huge level of phenotypic variation between species, even within the mammalian or avian classes, we must assume this diversity comes from distinctions at the protein level. Our analysis here, and elsewhere (6), has emphasized the diversity found between the proteomes of various species, specifically at the level of the phosphoproteome, which is our focus. Chicken and turkey are two relatively closely related species; their genome similarities exhibit this despite approximately 40 million years of species divergence (21). However, our proteinto-protein comparison showed that there is greater divergence at the protein level, as even orthologous proteins between the two species were on average only $83.28 \%$ identical. This result alone highlights what poultry researchers, based on their own first-hand experience, have known: turkeys are not simply "big chickens."
The level of distinction in the proteome is also observed within the phosphorylation target sites recognized by kinases. In a phosphosite-to-phosphosite comparison, which represents well over 200,000 phosphorylation target sequences, approximately 70 $75 \%$ were found as exact matches in both the chicken and turkey proteomes (Table 3). In comparison, when comparing the human peptide sequences to that of our closest evolutionary relative, the chimpanzee, $82.98 \%$ were identical in both proteomes. This analysis suggests that using chicken as a model for the turkey may, in relative terms, be less valid than using chimp as a model for human. The level of divergence at these important enzymatic sites emphasizes the need for a turkey-specific approach to the study of this species. If this is the case for turkey and chicken, it is worth noting that between humans and mice the differences are more pronounced; there is only complete phosphorylation site sequence 
identity in $36.67 \%$ of peptides. Considering the sheer number of studies that utilize the mouse as a model for human physiology, it is worth taking into account that these species diverge so significantly at the phosphoproteome level. These differences highlight the need and the potential power of designing species-specific peptide arrays to study the kinomes of even closely related species.

We have described here three levels of comparison between the chicken and turkey: (1) genome, (2) orthologous protein, and (3) kinase recognition target site. At each of these levels, the relative similarity between chicken and turkey diminishes. While the genome level displays nearly $90 \%$ identity the kinase target sequence identity is between 70 and $75 \%$. This indicates that the phenotypic species divergence is greater at the proteome level and even greater still at this site of enzymatic activity.

Our group has conducted numerous studies on the physiology and specifically the host-pathogen interactions of several agricultural species (including chicken) using the species-specific peptide array technique. To date, we have focused on individual biological processes, such as metabolism or innate immunity. Our analysis here shows that the metabolism and immunity processes may be distinctions without a difference. Figure 1 highlights the interactions that are present between proteins that are part of these two putatively different biological functional groupings. This level of interaction was the impetus for our design of an immunometabolic, species-specific peptide array for chicken and turkey that we report here for the first time. In the realm of animal agriculture, nutrition/metabolism and immune performance have been two fields that have been converging for many years. Producers, veterinarians, and animal science researchers have come to understand that a sole focus on growth can often come at the expense of health and disease susceptibility, and a strong response to disease can have significant effects on growth (24). With a tool that can study both metabolism and immunity simultaneously, these two areas of animal science (and poultry production) no longer have to be at odds. We can study nutrition and observe effects on immune responses or, conversely, study disease and see how this may effect growth. Our group has already shown that a Salmonella infection of chicken can have effects on the fat deposition and carbohydrate metabolism in peripheral muscle (10). We look forward to the discovery that will be possible when the tool we have described is put to use on any number of nutritional-, metabolic-, and disease-related questions affecting the poultry industry and poultry health.

\section{AUTHOR CONTRIBUTIONS}

Ryan J. Arsenault conceived of the study, set out the analysis parameters, designed the final immuno-metabolic array, and wrote the paper. Brett Trost conducted the proteome comparisons and generated most of the data outputs. Michael $\mathrm{H}$. Kogut advised on analysis presentation and provided experimental resources. Ryan J. Arsenault and Brett Trost analyzed the data. Brett Trost and Michael H. Kogut reviewed and edited the paper.

\section{SUPPLEMENTARY MATERIAL}

The Supplementary Material for this article can be found online at http://www.frontiersin.org/Journal/10.3389/fvets.2014. $00022 /$ abstract
Supplemental Table S1 | Chicken immuno-metabolism design table. The table includes the human proteins and their chicken equivalents, along with the chicken-specific kinase target peptide sequences and the human annotations of the proteins.

Supplemental Table S2 | Turkey DAPPLE output of the equivalent proteins as on the chicken array. The table includes the DAPPLE output file for the turkey proteins to be included in the turkey-specific immuno-metabolism peptide array. Each protein to be incorporated into the array contains numerous peptide sequences which were Blast hits within the turkey proteome. These individual sequences are then selected for incorporation onto the peptide array. "Query" represents annotated database species and "Hit" represents species of interest: turkey.

\section{REFERENCES}

1. Arnold CN, Xia Y, Lin P, Ross C, Schwander M, Smart NG, et al. Rapid identification of a disease allele in mouse through whole genome sequencing and bulk segregation analysis. Genetics (2011) 187:633-41. doi:10.1534/genetics.110. 124586

2. Rubin CS, Rosen OM. Protein phosphorylation. Annu Rev Biochem (1975) 44:831-87. doi:10.1146/annurev.bi.44.070175.004151

3. Manning G, Whyte DB, Martinez R, Hunter T, Sudarsanam S. The protein kinase complement of the human genome. Science (2002) 298:1912-34. doi:10.1126/science.1075762

4. Zhang H, Zha X, Tan Y, Hornbeck PV, Mastrangelo AJ, Alessi DR, et al. Phosphoprotein analysis using antibodies broadly reactive against phosphorylated motifs. J Biol Chem (2002) 277:39379-87. doi:10.1074/jbc. M206399200

5. Mann M, Ong S, Grønborg M, Steen H, Jensen ON, Pandey A. Analysis of protein phosphorylation using mass spectrometry: deciphering the phosphoproteome. Trends Biotechnol (2002) 20:261-8. doi:10.1016/S0167-7799(02)01944-3

6. Arsenault R, Griebel P, Napper S. Peptide arrays for kinome analysis: new opportunities and remaining challenges. Proteomics (2011) 11(24):4595-609. doi:10.1002/pmic.201100296

7. Lowenberg M, Tuynman J, Scheffer M, Verhaar A, Vermeulen L, van Deventer S, et al. Kinome analysis reveals nongenomic glucocorticoid receptordependent inhibition of insulin signaling. Endocrinology (2006) 147:3555-62. doi:10.1210/en.2005-1602

8. Schrage YM, de-Bruijn IHB, de Miranda NFCC, van Oosterwijk J, Taminiau AHM, van Wezel T, et al. Kinome profiling of chondrosarcoma reveals Src pathway activity and dasatinib as option for treatment. Cancer Res (2009) 69:6216-22. doi:10.1158/0008-5472.CAN-08-4801

9. Arsenault RJ, Li Y, Maattanena P, Scruten E, Doig K, Potter A, et al. Altered toll-like receptor 9 signaling in Mycobacterium avium subsp. paratuberculosisinfected bovine monocytes reveals potential therapeutic targets. Infect Immun (2013) 81(1):226-37. doi:10.1128/IAI.00785-12

10. Arsenault RJ, Napper S, Kogut MH. Salmonella enterica typhimurium infection causes metabolic changes in chicken muscle involving AMPK, fatty acid and insulin/mTOR signaling. Vet Res (2013) 44(1):35. doi:10.1186/12979716-44-35

11. Arsenault RJ, Li Y, Bell K, Doig K, Potter A, Griebel PJ, et al. Mycobacterium avium subsp. paratuberculosis inhibits interferon gamma-induced signaling in bovine monocytes: insights into the cellular mechanisms of Johne's disease. Infect Immun (2012) 80(9):3039-48. doi:10.1128/IAI.00406-12

12. Arsenault RJ, Li Y, Potter A, Griebel PJ, Kusalik A, Napper S. Induction of ligandspecific PrPC signaling in human neuronal cells. Prion (2012) 6(5):477-88. doi:10.4161/pri.21914

13. Kindrachuk J, Arsenault R, Kusalik T, Kindrachuk KN, Trost B, Napper S, et al. Systems kinomics demonstrates congo basin monkeypox virus infection selectively modulates host cell signaling responses as compared to West African monkeypox virus. Mol Cell Proteomics (2011) 11(6):M111.015701. doi:10.1074/mcp.M111.015701

14. Jalal S, Arsenault R, Potter AA, Babiuk LA, Griebel PJ, Napper S. Genome to kinome: species-specific peptide arrays for kinome analysis. Sci Signal (2009) 2(54):11. doi:10.1126/scisignal.254pl1

15. Trost B, Arsenault R, Griebel P, Napper S, Kusalik A. DAPPLE: a pipeline for the homology-based prediction of phosphorylation sites. Bioinformatics (2013) 29(13):1693-5. doi:10.1093/bioinformatics/btt265 
16. Trost B, Kindrachuk J, Scruten E, Griebel P, Kusalik A, Napper S. Kinotypes: stable species- and individual-specific profiles of cellular kinase activity. BMC Genomics (2013) 14:854. doi:10.1186/1471-2164-14-854

17. Robertson AJ, Trost B, Scruten E, Robertson T, Mostajeran M, Connor W, et al. Identification of developmentally-specific kinotypes and mechanisms of varroa mite resistance through whole-organism, kinome analysis of honeybee. Front Genet (2014) 5:139. doi:10.3389/fgene.2014.00139

18. Arsenault RJ, Jalal S, Babiuk LA, Potter A, Griebel PJ, Napper S. Kinome analysis of toll-like receptor signaling in bovine monocytes. J Recept Signal Transduct Res (2009) 29(6):299-311. doi:10.3109/10799890903295127

19. Ritsema T, Joore J, van Workum W, Pieterse CJM. Kinome profiling of Arabidopsis using arrays of kinase consensus substrates. Plant Methods (2007) 3:3. doi:10.1186/1746-4811-3-3

20. Coers W, Timens W, Kempinga C, Klok PA, Moshage H. Specificity of antibodies to nitric oxide synthase isoforms in human, guinea pig, rat, and mouse tissues. J Histochem Cytochem (1998) 46:1385-91. doi:10.1177/002215549804601207

21. Dalloul RA, Long JA, Zimin AV, Aslam L, Beal K, Blomberg LeAnn, et al. Multi-platform next-generation sequencing of the domestic turkey (Meleagris gallopavo): genome assembly and analysis. PLoS Biol (2010) 8(9):e1000475. doi:10.1371/journal.pbio.1000475

22. Mathis D, Shoelson SE. Focus on: metabolism and immunology immunometabolism: an emerging frontier. Nat Rev Immunol (2011) 11:81-3. doi:10.1038/ nri2922

23. Finlay D, Cantrell DA. Metabolism, migration and memory in cytotoxic T cells. Nat Rev Immunol (2011) 11:109-17. doi:10.1038/nri2888

24. Humphrey BD, Klasing KC. Modulation of nutrient metabolism and homeostasis by the immune system. World Poul Sci J (2004) 60:90. doi:10.1079/WPS20037

25. Hornbeck PV, Kornhauser JM, Tkachev S, Zhang B, Skrzypek E, Murray B, et al. PhosphoSitePlus: a comprehensive resource for investigating the structure and function of experimentally determined post-translational modifications in man and mouse. Nucleic Acids Res (2012) 40:D261-70. doi:10.1093/nar/gkr1122

26. Waterhouse RM, Zdobnov EM, Tegenfeldt F, Li J, Kriventseva EV. OrthoDB: a hierarchical catalog of animal, fungal and bacterial orthologs. Nucleic Acids Res (2012) 41(Database issue):D358-65. doi:10.1093/nar/gks1116

27. Rice P, Longden I, Bleasby A. EMBOSS: the European molecular biology open software suite. Trends Genet (2000) 16:276-7. doi:10.1016/S0168-9525(00) 02024-2
28. Needleman SB, Wunsch CD. A general method applicable to the search for similarities in the amino acid sequence of two proteins. J Mol Biol (1970) 48:443-53. doi:10.1016/0022-2836(70)90057-4

29. The UniProt Consortium. Activities at the universal protein resource (UniProt). Nucleic Acids Res (2014) 42:D191-8. doi:10.1093/nar/gkt1140

30. Kreegipuu A, Blom N, Brunak S, Jarv J. Statistical analysis of protein kinase specificity determinants. FEBS Lett (1998) 430:45-50. doi:10.1016/S0014-5793(98) 00503-1

31. Chimpanzee Sequencing and Analysis Consortium. Initial sequence of the chimpanzee genome and comparison with the human genome. Nature (2005) 437:69-87. doi:10.1038/nature04072

32. Arsenault RJ, Kogut MH, He H. Combined CpG and poly I:C stimulation of monoctyes results in unique signaling activation not observed with the individual ligands. Cell Signal (2013) 25:2246-54. doi:10.1016/j.cellsig.2013.07.014

33. Franceschini A, Szklarczyk D, Frankild S, Kuhn M, Simonovic M, Roth A, et al. STRING v9.1: protein-protein interaction networks, with increased coverage and integration. Nucleic Acids Res (2013) 41:D808-15. doi:10.1093/nar/gks1094

Conflict of Interest Statement: The authors declare that the research was conducted in the absence of any commercial or financial relationships that could be construed as a potential conflict of interest.

Received: 16 April 2014; paper pending published: 08 May 2014; accepted: 29 October 2014; published online: 13 November 2014.

Citation: Arsenault RJ, Trost B and Kogut MH (2014) A comparison of the chicken and turkey proteomes and phosphoproteomes in the development of poultryspecific immuno-metabolism kinome peptide arrays. Front. Vet. Sci. 1:22. doi: 10.3389/fvets.2014.00022

This article was submitted to Veterinary Infectious Diseases, a section of the journal Frontiers in Veterinary Science.

Copyright $\odot 2014$ Arsenault, Trost and Kogut. This is an open-access article distributed under the terms of the Creative Commons Attribution License (CC BY). The use, distribution or reproduction in other forums is permitted, provided the original author(s) or licensor are credited and that the original publication in this journal is cited, in accordance with accepted academic practice. No use, distribution or reproduction is permitted which does not comply with these terms. 